\title{
The Role of the S\&P 500 Index Constituents in Tracking the U.S. Equity Market
}

\author{
Ebenezer Asem ${ }^{1} \&$ Shamsul Alam ${ }^{1}$ \\ ${ }^{1}$ Faculty of Management, University of Lethbridge, Lethbridge, Alberta, Canada \\ Correspondence: Ebenezer Asem, Faculty of Management, University of Lethbridge, Lethbridge, AB, T1K 3M4, \\ Canada. Tel: 1-403-382-7142. E-mail: ebenezer.asem@uleth.ca
}

Received: September 15, 2012

Accepted: September 29, 2012 Online Published: October 23, 2012

doi:10.5539/ijef.v4n12p15

URL: http://dx.doi.org/10.5539/ijef.v4n12p15

\begin{abstract}
We investigate how the S\&P committee balances the Index's goals of representing the U.S. equity market as well as capturing the performance of the equity market through its addition/deletion decisions. Our results show that the Index represents the US equity market very well by maintaining large stocks. However, these large stocks underperform the Index by $0.24 \%$ per month. The index committee makes up the lower performance of these stocks by adding high-performance stocks when deletions occur. The deletion/addition events are therefore crucial in both representing the equity market and tracking it performance.
\end{abstract}

Keywords: event studies, index performance, S\&P 500 constituents

\section{Introduction}

Introduced in 1957, the S\&P 500 Index (henceforth the Index) is regarded as the principal barometer of the US equities market and is the performance benchmark for many funds. (Note 1) Thus, the Index has the dual roles of representing the U.S. equity market (percentage of the equity market captured by the Index) and acting as a U.S. equity market performance benchmark. To act as an appropriate performance benchmark, the Index's performance should reflect the US equity market performance. However, the firms that capture the U.S. equity market (large firms) do not necessarily exhibit performance that is representative of the equity market performance. We study how the S\&P committee balances the dual goals of the Index in their addition/deletion decisions. For instance, a large firm may underperform the market, but the S\&P committee may retain the firm in the Index due to its size (representation of the US equity market).

Both representing the equity market and capturing its performance are influenced by the companies in the Index, which have not, and cannot, remain the same over long periods due to natural company occurrences such as bankruptcies, restructuring, mergers and acquisitions. In addition to these events, a firm can also be deleted from the Index if it substantially violates an inclusion threshold. (Note 2) Since its inception, roughly 1,600 companies have been deleted from the Index and the same number of companies has been added. We study how the S\&P committee balances the dual goals of the Index in its delete/add decisions. This is important since a large firm may not necessarily deliver performance that is representative of the U.S. equity market. We find that large firms survive in the Index despite their poor performance, underperforming U.S. equity market (and the Index) the Index by $0.24 \%$ per month. Thus, the Index's was able to track the U.S. equity performance by the additions of strong-performing stocks and deletions of smaller poor-performing stocks. This suggests that the addition/deletion events are critical for the Index in capturing the market's performance.

Stock additions to and deletions from the Index have attracted considerable research, primarily focusing on whether the abnormal returns associated with stock additions are consistent with information certification or with demand pressure. (Note 3) To our knowledge, only two studies compare the performance of the original 500 stocks to that of the updated Index to investigate whether the updating activities hinder or enhance the Index performance. Foster and Kaplan (2001) report that the updated 500 companies consistently generated higher returns than the original 500 companies that made up the Index in 1957. This result suggests that actively managing a portfolio to mimic the updated Index generates higher returns than buying and holding the original 500 companies. Thus, the study suggests that replacing the original stocks has enhanced the Index's performance. 
In contrast to Foster and Kaplan, Siegel and Schwartz (2006) report that the returns of the original 500 companies are significantly higher than those of the updated companies from 1957 through 2003. Siegel and Schwartz attribute Foster and Kaplan's contrary results to their focus on market values. In particular, updating the Index typically involves replacing lower-valued companies with higher-valued companies and this inflates the value of the continuously updated Index. Siegel and Schwartz report that, while the return based on market value is higher for the updated Index, the returns per share including reinvested dividends are higher for the original 500 stocks than the updated stocks. Siegel and Schwartz's finding suggests that replacing some of the original 500 stocks has hindered the Index's performance.

Unlike Foster and Kaplan (2001) and Siegel and Schwartz (2006), we do not focus on whether buying and holding the original 500 stocks represents a better investment strategy than investing in the updated Index, rather, we focus on how equity market representation and performance considerations influence the S\&P committee's add/delete stock decisions. To analyze this, we classify all stocks that were ever members of the Index by whether they survived in the Index or were deleted from the Index or were added to the Index. We analyze the performance and the market capitalizations of these portfolios to determine whether the stocks that survived in the Index did so because of their performance or because of their market capitalizations. In terms of performance, we first investigate whether the performance of the updated Index reflects the U.S. equity market performance by comparing the Index's return to the return of all stocks listed in the CRSP database. The results show that the Index's return mimics the market's return very well over our sample period.

Turning to analyzing the Index's performance, we find that the stocks that continuously survived in the Index underperform the Index (and the market). Also, the survived stocks did not outperform the deleted stocks. It is possible that this is due to the fact that some of the deletions occur due to natural firm events such as mergers or acquisitions or due to general changes to the Index such as the reorganization of the Index in 1976 to include the financial sector. Since these events are not entirely discretionary, the performance of the stocks that were dropped from the Index may not necessarily be inferior compared to that of those that were retained in the Index. As a result, we also compare the survived stocks to only the stocks that were voluntarily deleted and the conclusions remain the same. Collectively, the results show that the stocks that survived in the Index underperform the updated Index and the equity market. Thus, the performance of the updated Index is representative of the equity market's performance mainly because of the strong performance of the added stocks. This suggests that addition/deletion events are vital in making the Index's performance representative of the equity market's performance.

Our finding that the stocks that were added to the Index enhanced the Index's performance, outperforming both the stocks that survived in the Index and those that were deleted from the Index, is not necessarily inconsistent with Siegel and Schwartz's (2006) finding that the original stocks outperformed the updated Index. Since the stocks that replaced the original stocks can also be replaced, and indeed these events occur frequently, the effect of deletions and additions on the Index's performance can be different from that implied from comparing the performances of the updated Index and the original stocks. (Note 4) Also, unlike Siegel and Schwartz's study, we do not focus on the performance of the stocks that were deleted from the Index after the deletion events and their study covers 1957 through 2003, while ours covers 1973 through 2009.

Since there is no evidence that the stocks that survived in the Index did so because of performance, we turn our attention to their market capitalization. In particular, if capturing the US equity market is important to the S\&P committee, they would retain large stocks to capture the US equity market and the performance of these stocks, as discussed earlier, might not necessarily be representative of the market's performance. Over our sample period, 100 stocks continuously survived in the Index and these stocks account for about a half of the Index's capitalization. This suggests that the survived stocks remained in the Index mainly due to equity market representation considerations and not because their performance was representative of the market's performance. In summary, we find that the S\&P committee maintains the Index's role of capturing the U.S. equity market by retaining stocks with high capitalizations and of capturing the equity market performance by replacing smaller non-performing stocks with high performance stocks.

Our study is of empirical relevance since stock additions/deletions can influence both the Index's representation of the U.S. equity market and its ability to mimics the performance of the equity market. In particular, if the Index's performance does not track the equity market well, it will not be an appropriate benchmark for evaluating the performance of investments (mutual funds, etc) if the U.S. equity market performance is deemed as the appropriate standard. Thus, it is essential to examine the ability of the Index to track the U.S. equity market and factors that influence this ability. Our finding that addition/deletion decisions have, in fact, help the 
Index to track the equity market performance is important in validating the use of the Index's performance as a benchmark for investments.

\section{Data}

Data on monthly constituents of the firms in the Index are obtained from the Compustat database and monthly stock returns are from the Center for Research on Stock Prices (CRSP) database. Compustat information on all 500 stocks in the Index starts from January 1973. (Note 5) Our sample period starts from January 1970 through December 2009. Since we examine stock returns three years after the delete/add events, our sample includes these events up to December 2006. (Note 6)

Figure 1 shows the distribution of delete/add events by year over our sample period. The figure shows that these events are not uniform over time and they vary from a high of 60 delete/add events in 1976 to a low of seven events in 1992. The total number of delete/add events over our sample period is 787 . Over this period, there are 1,254 different firms that were members of the Index at one point or another. Only 100 of the 500 stocks in January 1973 remained in the Index over our entire sample period and 112 of these stocks were in the Index in December 2006, suggesting 12 of them were deleted and readmitted into the Index. Table 1 provides a description of stock deletion and addition events from January 1973 through December 2006. A total of 769 distinct stocks were deleted and 782 distinct stocks were added to the Index. Thus, there are more repeated deletions than repeated additions. Since 400 of the deletions were stocks in January 1973, 369 (769-400) of the "new" $770(782-12)$ stock additions over the sample period were deleted in the same period. Thus, almost a half of the stocks that were added to the Index over the sample period were deleted.

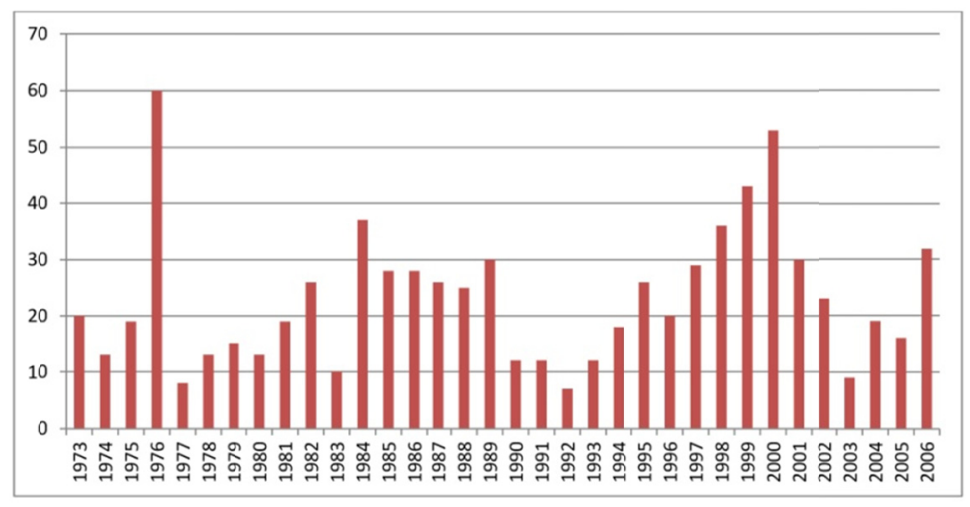

Figure 1. Number of deletions/additions from the S\&P 500 index by year

Table 1. Changes to the S\&P 500 Index Constituents

\begin{tabular}{lccccc}
\hline & Deletion Events & & \multicolumn{2}{c}{ Addition Events } \\
Frequency of Event & 1 & 2 & 3 & 1 & 777 \\
\hline Number of Stocks & 752 & 16 & 1 & 777 & 5 \\
Total & 752 & 32 & 3 & 10 \\
\hline
\end{tabular}

The table shows the frequency of stock deletions and additions to the S\&P 500 Index from January 1973 through December 2006.

Of the 769 distinct deletions, 592 were also delisted from the CRSP database by the end of 2006. In line with previous studies (e.g., Siegel and Schwartz, 2006), we use delisting reasons from CRSP to gauge the reasons for deletions from the Index. If deletion from CRSP occurs more than six months after the Index deletion, we assume that the Index deletion was discretionary. Of the 592 deletions from CRSP, 452 occurred within six months of the Index deletion. Among these, 424 were due to mergers, 20 to acquisitions, five to bankruptcies, and the remaining three to liquidation, insufficient market makers or delinquency in filing exchange reports.

\section{Methodology}

We focus on how the S\&P committee balances the dual goals of the Index to represent the equity market and achieve a performance that is representative of the equity market's performance in its add/delete decisions. To analyze this, we partition all stocks that were ever part of the Index in our sample period into three portfolios: (i) 
the portfolio of stocks that survived in the Index throughout our sample window, (ii) the portfolio of all stocks that were deleted from the Index, and (iii) the portfolio of all stocks that were added to the Index, and study the performance and market capitalization of these portfolios.

We first investigate the performance of the survived stocks to determine whether they were able to maintain their membership in the Index because of their performance. We use the average returns of the stocks in the portfolios to gauge the performance of the portfolios. In particular, we analyze the value-weighted, the equally-weighted, and the risk-adjusted returns of our portfolios. The risk-adjusted return is computed as the return scaled by the standard deviation and, thus, we control for total risk. If the stocks that survived in the Index retained their membership because of their performance, then these stocks should not underperform the updated Index and they should outperform those that were deleted from the Index. That is, if stocks were deleted due to poor performance, then the stocks that were deleted should clearly underperform those that survived in the Index. Some of the deletions that occurred were non-discretionary and, hence, we also compare the performance of the stocks that survived in the Index against that of those that the S\&P committee deleted at its discretion. In summary, we test the mean return of the firms that survived in the Index against those of the updated stocks, the deleted stocks, and voluntarily deleted stocks to ascertain the importance of performance in retaining the survived stocks using t-tests for the means and Wilcoxon's rank tests for the medians.

Next, we study whether market representation (capitalization) is important in the S\&P committee's add/delete decisions by investigating the capitalization of the survived portfolio. If equity market representation is important to the S\&P committee, it will tend to retain stocks with high capitalization although they may not be high performers. To analyze the market capitalization of the survived stocks, we calculate the proportion of the survived stocks' capitalization to the Index's capitalization each month and test whether the mean ratio is different from the relative number of the survived stocks. That is we test: (capitalization of survived stocks/Index's capitalization $)=($ number of stocks that survived/500). If the S\&P committee tends to keep stocks with high capitalization, the mean relative capitalization of the survived stocks should be greater than their relative number in the Index.

\section{Performance versus Capitalization in Add/Delete Decisions}

We first analyze whether the performance of the Index mimics that the performance of the US equity market and whether the Index captures the U.S. equity market. We proxy the U.S. equity market by all NYSE, AMEX, and NASDAQ common stocks listed in the CRSP database and, hence, we gauge the market's return by the value-weighted return of these stocks. Figure 2 plots the yearly returns on the U.S. equity market and S\&P 500 stocks and it shows that the return on the S\&P 500 index tracks the equity market return well. Also, the monthly returns show that return on the Index mimics the return on the CRSP index very well with a Pearson correlation coefficient of 0.983 and the geometric and arithmetic average returns including dividends for the CRSP index over the period is 1.22 and monthly 1.27 (compare with 1.21 and 1.26 for the S\&P Index). These suggest that the Index tracks the U.S. equity market performance very well over our sample period. Figure 3 presents the yearly percentage of the U.S. equity market capitalization that is captured by the Index's capitalization. The results show that the S\&P 500 stocks capture a large percentage of the U.S. equity market capitalization. This percentage increases from about $70 \%$ in the initial years to about $80 \%$ in the final years. Over the sample period, the S\&P 500 stocks capture an average of $73 \%$ of the U.S. equity market, indicating the Index is a good representation of the U.S. equity market.

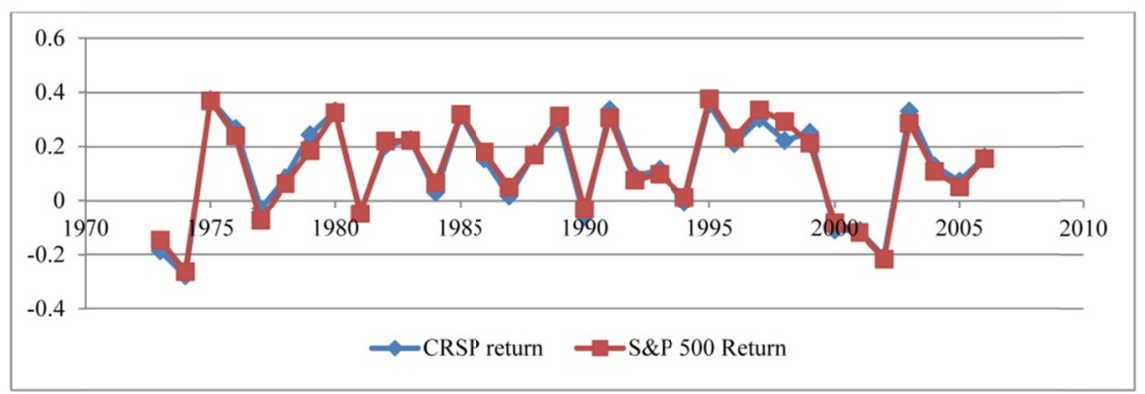

Figure 2.

The figure reports the yearly value-weighted return on all NYSE, AMEX, and NASDAQ common stocks that are listed in the CRSP and the returns of the stocks in the S\&P 500 Index from 1973 through 2006. 
We next turn to analyzing how the S\&P committee achieves the dual goals of representing the U.S. equity market and mimicking the market's performance by focusing on the stocks that continuously survived in the Index. We first analyze the contribution of these stocks to the Index's performance to determine whether they survived in the Index because their performance reflects the performance of the Index and, therefore, the U.S. equity market. Panel A of Table 2 presents the average value-weighted returns and Panel B reports the equally-weighted results for the S\&P updated portfolio, the survived portfolio, the added stock portfolio, the deleted stock portfolio and the discretionary deleted stock portfolio. The average returns for all portfolios, including the deleted portfolio, are based on the return when the stocks in the portfolios were members of the Index. From the results, we see that the stocks that continuously survived in the Index do not outperform the updated Index. For instance, the geometric mean value-weighted return is $1.21 \%$ per month for the updated Index, while it is $0.97 \%$ for the stocks that continuously survived in the Index. In addition, we see that the stock that continuously survived in the Index do not outperform the stocks that were deleted from the Index. For example, the geometric mean return of the stocks that were deleted is $1.11 \%$ and that for discretionary deletions is $1 \%$ (the results based on the arithmetic average return or the median return are similar).

Table 2. The Returns of the S\&P 500 stocks, the Continuously Survived Stocks, the Deleted Stocks and the Added Stocks

\begin{tabular}{lcccr}
\hline \multicolumn{1}{c}{ Portfolio } & $\begin{array}{c}\text { Geometric Mean } \\
\text { Return }\end{array}$ & $\begin{array}{c}\text { Arithmetic Mean } \\
\text { Return }\end{array}$ & $\begin{array}{c}\text { Risk-Adjusted } \\
\text { Arithmetic Return }\end{array}$ & Median Return \\
\hline Updated Stock Portfolio (1) & \multicolumn{1}{c}{ Panel A: } & Value-Weighted Returns & & \\
Survived Stock Portfolio (2) & 1.21 & $1.26^{2,3,5}$ & 0.39 & $1.34^{2,3,5}$ \\
Added Stock Portfolio (3) & 0.97 & $1.05^{3,4}$ & 0.26 & $1.0026^{3,4}$ \\
Deleted Stock Portfolio (4) & 1.62 & $1.62^{4,5}$ & 0.76 & $1.66^{4,5}$ \\
Discretionary Deleted Stocks (5) & 1.11 & 1.21 & 0.35 & 1.17 \\
& 1.00 & 1.07 & 0.29 & 1.01 \\
Updated Stock Portfolio (1) & Panel B: & Equally-Weighted Returns & & $1.35^{2,3,5}$ \\
Survived Stock Portfolio (2) & 1.06 & $1.10^{2,3,5}$ & 0.41 & $0.98^{3,4}$ \\
Added Stock Portfolio (3) & 0.80 & $0.89^{3,4}$ & 0.22 & $1.31^{4,5}$ \\
Deleted Stock Portfolio (4) & 1.21 & $1.22^{4,5}$ & 0.85 & 1.09 \\
Discretionary Deleted Stocks (5) & 0.93 & 0.97 & 0.34 & 0.90 \\
\hline
\end{tabular}

The table shows the average monthly percentage returns for the firms in the S\&P 500 Index, those that continuously survived, those that were added to and those that were deleted from the Index from January 1973 through December 2006. The returns are for only the months that the stocks were actually in the Index.

${ }^{2,3,4,5}$ Indicate that the mean is different from the mean in portfolio $2,3,4,5$ at the $5 \%$ level of test, respectively. We use t-tests for the difference in means and Wilcoxon's signed rank tests for the difference in medians.

It is possible that the stocks that were deleted from the Index performed poorly in the immediate periods preceding the deletion events. Consequently, we report the returns of the stocks that were deleted in the one, two and three years prior to the deletion events in Table 3. For completeness, we also report the returns of the added stocks prior to the addition events. The results show that firms that were deleted from the Index were, in fact, losing value in the years preceding the deletion events (the average returns of the deleted stocks and the voluntary deleted stocks are negative). Clearly, the deleted stocks underperformed the survived stocks in the one to three years prior to the deletion events. However, we also find that 88 of the 100 stocks that survived also had one- two- and three-year returns that were lower than the geometric average return of the firms that were voluntarily deleted at some points over our sample period, but they have remained in the Index. Thus, it does not appear that the firms that survived in the Index did so because their performance was representative of the equity market performance. As a result, we turn our attention to investigating the capitalizations of the survived stocks.

Each month, we compute the capitalization of the 100 firms that continuously survived in the Index and scale it by the capitalization of all the 500 firms in the Index. Figure 3 reports the average yearly ratios over our sample period and the results show that this ratio is about a half (the average over our sample period is 0.502 ). That is, the 100 stocks that survived in the Index account for half of the Index's capitalization. T-test shows that the mean capitalization of the 100 stocks in the Index (0.502) is larger than the proportion of their number in the 
Index (0.2) with p-value less than 0.00 . Thus, it appears that the stocks that consistently survived in the Index did so mainly because of their capitalization and not their performance.

Table 3. The Returns of Stocks before the S\&P 500 Index Delete/Add Events

\begin{tabular}{lccc}
\hline \multicolumn{1}{c}{ Months Before Events } & -37 to -1 & -25 to -1 & -13 to -1 \\
\hline & Panel A: Mean Value-Weighted Returns & & $1.77^{2,3}$ \\
Additions (1) & $1.55^{2,3}$ & $1.63^{2,3}$ & $-1.30^{3}$ \\
Deletions (2) & -0.89 & $-1.32^{3}$ & -1.69 \\
Discretionary Deletions (3) & -1.07 & -1.67 & $1.95^{2,3}$ \\
& Panel B: Mean Equally-Weighted Returns & & $-1.30^{3}$ \\
Additions (1) & $1.82^{2,3}$ & $1.87^{2,3}$ & -1.64 \\
Deletions (2) & -0.78 & $-1.27^{3}$ & -1.59 \\
Discretionary Deletions (3) & -0.98 & & $1.56^{2,3}$ \\
& Panel C: Value Weighted Returns & 0.13 \\
Additions (1) & $1.45^{2,3}$ & $1.49^{2,3}$ & -0.45 \\
Deletions (2) & -0.56 & -0.54 & -0.73 \\
Discretionary Deletions (3) & -0.66 & & $15.36^{2,3}$ \\
& Panel D: Value Weighted Returns & $-3.34^{2}$ \\
Additions (1) & $18.87^{2,3}$ & $-5.97^{2,3}$ & -5.98 \\
Deletions (2) & $-2.45^{2}$ & $-4.76^{2}$ & -6.32 \\
Discretionary Deletions (3) & -4.55 & & \\
\hline
\end{tabular}

The table shows the mean monthly percentage returns of the stocks that were added, deleted, and voluntarily deleted from the S\&P 500 Index from 1973 through 2006. Since our longest window of our event analysis is three years, our sample covers 1970 through 2009. Panel A presents the mean value-weighted returns for the 12 months, 24 months and 36 months prior to and subsequent to the event months. Panel B, $\mathrm{C}$, and D present the results for the equally weighted returns, the median returns and the risk-adjusted returns (returns scaled by the standard deviation), respectively. P-values based on t-tests for the means and Wilcoxon's signed rank tests for the medians are reported in parentheses.

Collectively, we find no evidence that the stocks that survived in the Index did so due to their performance. In fact, the survived stocks underperform both the Index and the U.S. equity market. In contrast, there is strong evidence that the survived stocks capture a large part of the Index's capitalization and, therefore, likely remained in the Index due to their equity market representation. It appears the S\&P committee makes up for the deficiency in the performance of the survived stocks by deleting smaller non-performing stocks and replacing them with high performance stocks. In fact, we see that the stocks that were added to the Index do not only display a strong performance when they became members of the Index, they also exhibit strong performance prior to the addition events, achieving a growth rate of over $1.4 \%$ per month in the one to three years prior to the additions (Table 3 ). Thus, the S\&P committee replaces smaller stocks that it observes are performing poorly with stocks that it observes are performing well.

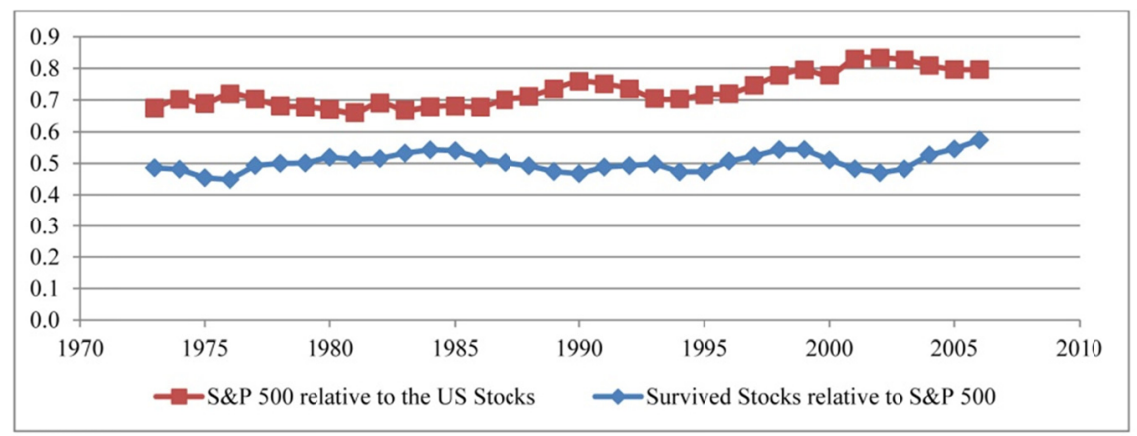

Figure 3.

The figure presents the dynamics of the percentage of the U.S. equity market (NYSE, AMEX, and NASDAQ) capitalization that is captured the S\&P 500 stocks and the percentage of the capitalization of the S\&P 500 stocks that is captured by 100 stocks that survived from 1973 through 2006. 


\section{Conclusion}

We investigate how the S\&P committee balances the Index's goals of representing the U.S. equity market as well as capturing the performance of the equity market through its addition/deletion decisions. The goal of representing the U.S. equity market can be achieved by including largest stocks in the Index. However, the performance of the largest stocks may not necessarily reflect the performance of the U.S. equity market. To study this, we initially examine whether the S\&P 500 Index tracks the U.S. equity market. Next, we investigate the contributions of the stocks that continuously survived in the Index, those that were deleted from the Index, and those that were added to the Index to the performance of the Index. Lastly, we study the capitalizations of these portfolios.

The results show that the S\&P 500 Index mimics the U.S. equity market performance very well and, therefore, serves as an appropriate benchmark for the performance of the U.S. equity market. However, we find that the contributions of the portfolios of survived stocks, deleted stocks, and added stocks to the Index's performance are different. In particular, our results show that the stocks that survived in the Index underperform the updated Index by $0.24 \%$ per month. In addition, the contribution of the survived stocks to the Index's performance is not different from that of the stocks that were deleted from the Index. Thus, based on performance alone, there is no reason why the stocks that survived in the Index should do so. It appears that the Index committee makes up for the short-fall in the performance of the survived stocks by adding high-performance stocks.

Finding no evidence that the stocks that survived in the Index did so because their performance was representative of the U.S. equity market, we investigate the capitalization of the survived portfolio. Over our sample period, one hundred stocks continuously survived in the Index. These stocks, which represent only $20 \%$ of the stocks in the Index, capture one half of the Index's capitalization. Thus, these survived stocks are relatively large compared to the deleted or added stocks. Overall, our results suggest the survived stocks were retained in the Index mainly because they represent the U.S. equity market and not because their performance reflects the equity market performance. Thus, we conclude that the S\&P committee capture the U.S. equity market by retaining stocks with large capitalizations in the Index and offsets their negative effect on the Index's performance by replacing smaller non-performing stocks with high-performing stocks.

Overall, our results show that the S\&P 500 Index captures the U.S. equity market well and its performance also mimics the U.S. equity market. However, its ability to play these dual roles is largely dependent on the S\&P committee's addition/deletion decisions. It is therefore essential to examine the Index's representation of the equity market as well as its ability to track the equity market performance if the Index is to remain an appropriate performance benchmark for evaluating the performance mutual funds and other investments. Overall, our findings suggest that the Index's performance is an appropriate benchmark for evaluating the performance of investments if the performance of the U.S. equity market is an appropriate standard.

\section{Acknowledgement}

We gratefully acknowledge financial support from the University of Lethbridge CREDO Fund. Any mistakes are ours.

\section{References}

Beneish, D. M., \& Whaley, R. E. (1996). An Anatomy of the S\&P Game: The Effects of Changing the Rules. Journal of Finance, 51, 1909-1930. http://dx.doi.org/10.2307/2329543

Beneish, D. M., \& Whaley, R. E. (2002). S\&P 500 Index Replacements. Journal of Portfolio Management, 29 , 51-60. http://dx.doi.org/10.3905/jpm.2002.319863

Blume, E. M., \& Edelen, R. M. (2004). S\&P 500 Indexers, Tracking Error, and Liquidity. Journal of Portfolio Management, 30, 37-46. http://dx.doi.org/10.3905/jpm.2004.412317

Elliot, W., Van Ness, B. F., Walker, M. D., \& Warr, R. (2006). What Drives the S\&P 500 Inclusion Effect? Analytical Survey. Financial Management, $31-48$. http://dx.doi.org/10.1111/j.1755-053X.2006.tb00158.x

Foster, R., \& Kaplan, S. (2001). Creative Destruction: Why Companies That Are Built to Last Underperform the Market - And How To Successfully Transform Them. New York: Random House.

Harris, L., \& Gurel, E. (1986). Price and Volume Effects Associated with Changes in the S\&P List: New Evidence for the Existence of Price Pressure. Journal of Finance, $\quad 41, \quad 815-829 . \mathrm{http}: / / \mathrm{dx}$. doi.org/10.1111/j.1540-6261.1986.tb04550.x 
Lynch, W. A., \& Mendenhell, R. (1997). New Evidence on the Stock Price Effects Associated with Changes in the S\&P 500 Index. Journal of Business, 70, 351-383. http://dx.doi.org/10.1086/209722

Shleifer, A. (1986). Do Demand Curves for Stocks Slope Down? Journal of Finance, 41, 579-590. http://dx.doi.org/10.1111/j.1540-6261.1986.tb04518.x

Siegel, J. J., \& Schwartz, J. D. (2006). Long-Term Returns on the Original S\&P 500 Companies. Financial Analysts Journal, 62, 18-31. http://dx.doi.org/10.2469/faj.v62.n1.4055

\section{Notes}

Note 1. In fact, the history of the Index dates back to 1923 when Standard and Poor's constructed the stock price index of all traded stocks. In 1926, a composite index was constructed with 90 stocks. Later, on March 4, 1957, the composite index was expanded to include 500 companies and the name was changed to the S\&P500 Index.

Note 2. To be included the company must (i) be a U.S. Company (set and implemented since 2002), (ii) have market capitalization in excess of US $\$ 5$ billion, (iii) have a public float of at least $50 \%$, (iv) be financially viable and have positive reported earnings for at least four consecutive quarters, (v) have adequate liquidity and reasonable price (the ratio of annual dollar value traded to market capitalization for the company to be 0.3 or greater), (vi) have sector representation for a diversified and representative portfolio (10 sectors in total), and (vii) be an operating company (however, some real-estate-investment trust and business-development companies are eligible for inclusion). For details, see www.indices.standardandpoors.com.

Note 3. Examples of these include Harris and Gruel (1986), Shleifer (1986), Beneish and Whaley (1996, 2002), Lynch and Mendenhell (1997), and Blume and Edelen (2004). Elliot, Van Ness, Walker and Warr (2006) provide a detailed survey of these studies.

Note 4. For example, suppose stock A is the only original stock that is replaced in the Index by stock B. Then a stronger performance of the original stocks than the updated stocks will imply that the performance of A after its deletion is stronger than B after its addition (i.e., the delete/add decision hinders the updated stocks' performance). If, however, $\mathrm{B}$ is also replaced by $\mathrm{C}$, then a stronger performance of the original stocks does not necessarily imply that the delete/add decisions hinder the performance of the updated stocks. In particular, if B performed poorly after its deletion, the performance of A and B when they were deleted can be worse than the performance of $\mathrm{B}$ and $\mathrm{C}$ when they were in the Index (i.e., the delete/add decisions did not hinder the updated stocks' performance). In other words, while the first delete/add decision was bad, the second one was good and this good event can overcompensate for the original bad decision.

Note 5. The number of stocks reported by Compustat for December 1972 is 495 and this number declines for earlier dates.

Note 6. Prior to 1976, there were 425 industrial firms, 25 retail and railroads, and 50 utilities. In 1976, 40 financial firms were added and industrial firms were reduced to 400 , retail and railroads to 20 , and utilities to 40 . 